David Preiss, Department of Mathematics, University College London, London WC1E 6BT, United Kingdom. e-mail: dp@math.ucl.ac.uk Luděk Zajíček, Department of Math. Anal., Charles University, Sokolovská 83, 18600 Prague 8, Czech Republic.

e-mail: zajicek@karlin.mff.cuni.cz

\title{
ON DINI AND APPROXIMATE DINI DERIVATES OF TYPICAL CONTINUOUS FUNCTIONS
}

\begin{abstract}
In the thirties, Banach, Mazurkiewicz and Jarník found relations connecting Dini derivates of a typical continuous function on $[0,1]$ at all points of $(0,1)$. We prove, answering a question of K. M. Garg, that there are no further relations of this sort. An analogous result is proved also for approximate Dini derivates. The aim of this note is to present relatively simple proofs of these results. An article containing an improvement of these results in several directions (with a considerably more complicated proof) is in preparation.
\end{abstract}

\section{Introduction}

In what follows we denote by $\mathcal{C}$ the space of all continuous real functions on $[0,1]$ equipped with the supremum norm $\|\cdot\|$. We put $\mathbb{R}^{*}=\mathbb{R} \cup\{-\infty, \infty\}$.

As usual, we say that the typical function in $\mathcal{C}$ has a property if all functions from a residual subset of $\mathcal{C}$ (i.e., all functions of $\mathcal{C}$ except a first category set) have this property.

The Dini derivates and the approximate Dini derivates of $f$ at $x$ are denoted by $D^{+} f(x), D^{-} f(x), D_{+} f(x), D_{-} f(x)$ and by $\bar{D}_{a p}^{+} f(x), \bar{D}_{a p}^{-} f(x), \underline{D}_{a p}^{+} f(x)$, $\underline{D}_{a p}^{-} f(x)$, respectively.

It is known that, for a typical function $f \in \mathcal{C}$, at every point $x \in(0,1)$ the following relations hold:

Key Words: typical continuous function, Dini derivate, Dini approximate derivate

Mathematical Reviews subject classification: 26A24

Received by the editors July 6, 1999

* Supported by CEZ J13/98113200007, GAČR 201/97/1161 and GAUK 160/1999 
(i) $([\mathrm{B}],[\mathrm{M}]) \max \left(\left|D^{+} f(x)\right|,\left|D_{+} f(x)\right|\right)=\max \left(\left|D^{-} f(x)\right|,\left|D_{-} f(x)\right|\right)=\infty$,

(ii) $([\mathrm{J} 1])\left[D_{-} f(x), D^{-} f(x)\right] \cup\left[D_{+} f(x), D^{+} f(x)\right]=\mathbb{R}^{*}$.

It is easy to see that these results can be reformulated as follows:

Theorem 1.1. ([B],[M], [J1]) For a typical function $f \in \mathcal{C}$, for every point $x \in(0,1)$ either

(a) $D^{+} f(x)=\infty, D_{-} f(x)=-\infty$ and $D_{+} f(x) \leq D^{-} f(x)$

or

(b) $D_{+} f(x)=-\infty, D^{-} f(x)=\infty$ and $D_{-} f(x) \leq D^{+} f(x)$.

A natural problem (cf. [G1], Remark 2 and [G2], p. 466) arises, whether there are other relations of this sort. The following theorem gives a negative answer to this question. It was proved by the first named author almost twenty years ago (cf. [Z1], where this result and its improvements are stated without a proof).

Theorem 1.2. Let $D^{+}, D_{+}, D^{-}, D_{-}$be extended real numbers such that either

$$
\text { (a) } D^{+}=\infty, \quad D_{-}=-\infty \text { and } D_{+} \leq D^{-}
$$

or

$$
\text { (b) } D_{+}=-\infty, \quad D^{-}=\infty \text { and } D_{-} \leq D^{+} \text {. }
$$

Then, for a typical $f \in \mathcal{C}$, there exists $x \in(0,1)$ such that

$$
D^{+}=D^{+} f(x), D_{+}=D_{+} f(x), D^{-}=D^{-} f(x), D_{-}=D_{-} f(x) .
$$

We note that only the cases

$$
D^{+}=\infty, D_{-}=-\infty, \quad D_{+}<D^{-} \text {and }\left\{D_{+}, D^{-}\right\} \neq\{-\infty, \infty\}
$$

and

$$
D_{+}=-\infty, D^{-}=\infty, D_{-}<D^{+} \text {and }\left\{D_{-}, D^{+}\right\} \neq\{-\infty, \infty\}
$$

are new (cf. [G1]). Indeed, the three remaining cases are covered by the following:

The case $D^{+}=D^{-}=\infty, D_{+}=D_{-}=-\infty$ follows from the well-known result of Jarník ([J1], cf. [Z1] for further improvements and generalizations) that, for a typical $f \in \mathcal{C}$, almost all point $x \in(0,1)$ are knot points of $f$, i.e., $D^{+} f(x)=D^{-} f(x)=\infty$ and $D_{+} f(x)=D_{-} f(x)=-\infty$.

The case $D^{+}=D_{+}=D^{-}=\infty, D_{-}=-\infty$ (and the three symmetrical cases) is covered by the well-known result of Saks [S]. 
The case $D^{+}=\infty, D_{-}=-\infty, D_{+}=D^{-}=r \in \mathbb{R}$ (and the three symmetrical cases) is due to Garg ([G1], Theorem 1, (iii)).

We will also show the approximate analogues of the above results; these were stated without proof in [Z1](p. 106, Theorem 3). The following approximate analogue of Theorem 1.1 easily follows from [J2] and [Z2].

Theorem 1.3. For a typical function $f \in \mathcal{C}$, for every point $x \in(0,1)$ either

$(\alpha) \bar{D}_{a p}^{+} f(x)=\infty, \underline{D}_{a p}^{-} f(x)=-\infty \quad$ and $\underline{D}_{a p}^{+} f(x) \leq \bar{D}_{a p}^{-} f(x)$

or

( $\beta) ~ \underline{D}_{a p}^{+} f(x)=-\infty, \bar{D}_{a p}^{-} f(x)=\infty$ and $\underline{D}_{a p}^{-} f(x) \leq \bar{D}_{a p}^{+} f(x)$.

Proof. The results of [J2] (cf. [Z1], p. 104, Theorem J, for the formulation of less known Jarník's results on typical continuous functions) immediately imply that, for a typical $f \in \mathcal{C}$, for each $x \in(0,1)$

$$
\begin{gathered}
\max \left(\left|\bar{D}_{a p}^{+} f(x)\right|,\left|\underline{D}_{a p}^{+} f(x)\right|\right)=\max \left(\left|\bar{D}_{a p}^{-} f(x)\right|,\left|\underline{D}_{a p}^{-} f(x)\right|\right)=\infty \text { and } \\
\{\infty,-\infty\} \subset\left\{\bar{D}_{a p}^{+} f(x), \underline{D}_{a p}^{+} f(x), \bar{D}_{a p}^{-} f(x), \underline{D}_{a p}^{-} f(x)\right\}
\end{gathered}
$$

and therefore either $\bar{D}_{a p}^{+} f(x)=\infty, \underline{D}_{a p}^{-} f(x)=-\infty$ or $\underline{D}_{a p}^{+} f(x)=-\infty$, $\bar{D}_{a p}^{-} f(x)=\infty$.

Theorem 4 (i) of [Z1], p. 106, (which was proved in [Z2]) immediately implies that, for a typical $f \in \mathcal{C}, \underline{D}_{a p}^{+} f(x) \leq \bar{D}_{a p}^{-} f(x)$ and $\underline{D}_{a p}^{-} f(x) \leq \bar{D}_{a p}^{+} f(x)$ for each $x \in(0,1)$. The statement of the theorem now follows from the fact that the union of two first category sets is of the first category.

Both Theorem 1.2 and its approximate analogue are special cases of the following theorem.

Theorem 1.4. Let $D^{+}, D_{+}, D^{-}, D_{-}$be extended real numbers such that either

(a) $D^{+}=\infty, D_{-}=-\infty$ and $D_{+} \leq D^{-}$

or

$$
\text { (b) } D_{+}=-\infty, D^{-}=\infty \text { and } D_{-} \leq D^{+} \text {. }
$$

Then, for a typical $f \in \mathcal{C}$, there exists $x \in(0,1)$ such that

$$
\begin{aligned}
& D^{+}=D^{+} f(x)=\bar{D}_{a p}^{+} f(x), D_{+}=D_{+} f(x)=\underline{D}_{a p}^{+} f(x), \\
& D^{-}=D^{-} f(x)=\bar{D}_{a p}^{-} f(x), D_{-}=D_{-} f(x)=\underline{D}_{a p}^{-} f(x) .
\end{aligned}
$$


The rest of the article is devoted to presenting a relatively simple proof of Theorem 1.4. An article containing its improvement in several directions is in preparation. These more complicated results (with much more complicated proofs) also contain the previous improvement of Theorem 1.4 announced without proof in [Z1] (Theorem 2 on page 106). The basic idea of the proof of the stronger results in this direction is the same as the one used here to show Theorem 1.4, but several rather technical refinements are also needed.

In order to include Saks's result [S] in the present development fully, Theorem 1.4 should assert that there exists a $c$-dense set $M$ of points $x$ with the desired property. In fact, a slight standard refinement of the present proof suffices to show this. Nevertheless, we do not do it, since the article in preparation will give (among other things) a satisfactory answer to the question how big the set $M$ is.

\section{Proofs}

To prove Theorem 1.4, we will need a number of lemmas. We will use the following notation.

The open ball in a metric space with the center $c \in X$ and the radius $r>0$ is denoted by $B(c, r)$.

If $M \subset \mathbb{R}$, then $|M|$ denotes the Lebesgue measure of $M$.

Definition 2.1. Let $\lambda^{+}<\lambda^{-}, 0<\tau<1$ be real numbers, $J=\left[y_{1}, y_{4}\right], I=$ $\left[y_{2}, y_{3}\right]$ be closed intervals and $f \in \mathcal{C}$. We say that $f$ is of type $A\left(\lambda^{+}, \lambda^{-}, J, I, \tau\right)$, if $0 \leq y_{1}<y_{2}<y_{3}<y_{4} \leq 1, y_{3}-y_{2}=\tau\left(y_{4}-y_{3}\right)=\tau\left(y_{2}-y_{1}\right)$ and $f$ is linear on the intervals $\left[y_{1}, y_{2}\right],\left[y_{2}, y_{3}\right],\left[y_{3}, y_{4}\right]$ with the slopes $\lambda^{-},\left(\lambda^{+}+\lambda^{-}\right) / 2, \lambda^{+}$, respectively.

Remark 1.. If $f$ is of type $A\left(\lambda^{+}, \lambda^{-}, J, I, \tau\right)$, then $J, I$ are concentric closed subintervals of $[0,1]$ and $|J|=\frac{2+\tau}{\tau}|I|$.

The following simple lemma is basic for the whole proof. Note that it can be easily generalized; for example, the equalities $y_{3}-y_{2}=\tau\left(y_{4}-y_{3}\right)=\tau\left(y_{2}-y_{1}\right)$ are not used in its proof.

Lemma 2.2. Let $g \in \mathcal{C}$ be of type $A\left(\lambda^{+}, \lambda^{-}, J, I, \tau\right), 0<\beta<(1 / 8)\left(\lambda^{-}-\right.$ $\left.\lambda^{+}\right)|I|$ and let $f \in B(g, \beta)$ be given. Then there exists $x \in \operatorname{int} I$ such that

$$
\frac{f(y)-f(x)}{y-x} \geq \lambda^{+} \quad \text { whenever } \quad x<y, y \in J
$$

and

$$
\frac{f(y)-f(x)}{y-x} \leq \lambda^{-} \quad \text { whenever } \quad y<x, y \in J
$$


Proof. Let $J=\left[y_{1}, y_{4}\right], I=\left[y_{2}, y_{3}\right]$. Put $s:=\left(y_{2}+y_{3}\right) / 2$. Consider the function $\psi$ on $\left[y_{1}, y_{4}\right]$ for which $\psi(s)=g(s)$ and which is linear on the intervals $\left[y_{1}, s\right],\left[s, y_{4}\right]$ with the slopes $\lambda^{-}, \lambda^{+}$, respectively. Clearly

$$
\lambda^{+} \leq \frac{\psi(y)-\psi(x)}{y-x} \leq \lambda^{-} \text {whenever } x, y \in\left[y_{1}, y_{4}\right], x \neq y
$$

and

$$
g(t)-\psi(t)=\frac{1}{4}\left(\lambda^{-}-\lambda^{+}\right)\left(y_{3}-y_{2}\right) \text { whenever } t \in\left[y_{1}, y_{2}\right] \cup\left[y_{3}, y_{4}\right]
$$

Put $m:=\min _{t \in\left[y_{1}, y_{4}\right]}(f(t)-\psi(t))$ and choose $x \in\left[y_{1}, y_{4}\right]$ for which $m=$ $f(x)-\psi(x)$. Since $f(s)-\psi(s)<\beta$, we have $m<\beta$. Since $m+\psi(x)=f(x)$ and $m+\psi(y) \leq f(y)$ for each $y \in\left[y_{1}, y_{4}\right]$, we easily see that (1) and (2) hold. Indeed, e.g. for $x<y \leq y_{4}$ we have (using also (3))

$$
\frac{f(y)-f(x)}{y-x} \geq \frac{(m+\psi(y))-(m+\psi(x))}{y-x}=\frac{\psi(y)-\psi(x)}{y-x} \geq \lambda^{+} .
$$

Further

$$
g(x)-\psi(x)=g(x)-f(x)+m<2 \beta<\frac{1}{4}\left(\lambda^{-}-\lambda^{+}\right)\left(y_{3}-y_{2}\right)
$$

and thus $x \in\left(y_{2}, y_{3}\right)$ by $(4)$.

The number $\tau$ plays an essential role in the following lemma and in its application.

Lemma 2.3. Let $g \in \mathcal{C}$ be of type $A\left(\lambda^{+}, \lambda^{-}, J, I, \tau\right), \beta>0, f \in B(g, \beta)$ and $x \in I$ be given. Then there exist numbers $0<h^{+}<|J|, 0<h^{-}<|J|$ such that

$$
\left|\left\{y \in\left(x, x+h^{+}\right):\left|\frac{f(y)-f(x)}{y-x}-\lambda^{+}\right|<\tau\left(\lambda^{-}-\lambda^{+}\right)+4 \beta /|I|\right\}\right| \geq h^{+} / 2
$$

and

$$
\left|\left\{y \in\left(x-h^{-}, x\right):\left|\frac{f(y)-f(x)}{y-x}-\lambda^{-}\right|<\tau\left(\lambda^{-}-\lambda^{+}\right)+4 \beta /|I|\right\}\right| \geq h^{-} / 2 .
$$

Proof. Let $J=\left[y_{1}, y_{4}\right], I=\left[y_{2}, y_{3}\right]$. Put $h^{+}=y_{4}-x$ and $K=[(x+$ $\left.\left.y_{4}\right) / 2, y_{4}\right]$. Choose an arbitrary $y \in K$. Since $\tau<1$, we have $y>y_{3}$ and therefore

$g(y)-g(x)=\frac{\lambda^{-}+\lambda^{+}}{2}\left(y_{3}-x\right)+\lambda^{+}\left(y-y_{3}\right)=\lambda^{+}(y-x)+\frac{\lambda^{-}-\lambda^{+}}{2}\left(y_{3}-x\right)$. 
Consequently

$\left|\frac{g(y)-g(x)}{y-x}-\lambda^{+}\right|=\frac{\lambda^{-}-\lambda^{+}}{2} \frac{y_{3}-x}{y-x} \leq \frac{\lambda^{-}-\lambda^{+}}{2} \frac{y_{3}-y_{2}}{\left(y_{4}-y_{3}\right) / 2}=\tau\left(\lambda^{-}-\lambda^{+}\right)$.

Since

$$
\left|\frac{f(y)-f(x)}{y-x}-\frac{g(y)-g(x)}{y-x}\right| \leq \frac{2 \beta}{\left(y_{4}-y_{3}\right) / 2} \leq \frac{4 \beta}{|I|},
$$

we obtain

$$
\left|\frac{f(y)-f(x)}{y-x}-\lambda^{+}\right| \leq \tau\left(\lambda^{-}-\lambda^{+}\right)+\frac{4 \beta}{|I|} .
$$

Since $|K|=h^{+} / 2$, this shows that $h^{+}$has the desired property. The existence of $h^{-}$can be proved in a symmetrical way.

The proofs of the following two almost obvious lemmas will be omitted.

Lemma 2.4. Let $\varphi \in \mathcal{C}, \gamma>0$ and $x \in(c, d) \subset[0,1]$ be given. Then there exists $\xi>0$ such that $[x-\xi, x+\xi] \subset(c, d)$ and

$$
\left|\frac{\varphi(y)-\varphi(x)}{y-x}-\frac{\varphi(y)-X^{*}}{y-x^{*}}\right|<\gamma,
$$

whenever $\left|x^{*}-x\right|<\xi,\left|X^{*}-\varphi(x)\right|<\xi$ and $y \in[0,1] \backslash(c, d)$.

Lemma 2.5. Let $\psi \in \mathcal{C}, \gamma>0$ and closed intervals $I, J$ such that $I \subset \operatorname{int} J \subset$ $(0,1)$ be given. Then there exists $\omega>0$ such that

$$
\left|\frac{f(y)-f(x)}{y-x}-\frac{\psi(y)-\psi(x)}{y-x}\right|<\gamma,
$$

whenever $f \in B(\psi, \omega), x \in I$ and $y \in[0,1] \backslash J$.

These two lemmas will be used to prove our last lemma.

Lemma 2.6. Let $\lambda^{+}<\lambda^{-}, \mu^{+}<\mu^{-}, x, \delta>0, \tau>0, \alpha>0$ be real numbers, $I, J$ be closed intervals and $\varphi \in \mathcal{C}$ be a piece-wise linear function such that $x \in$ int $I, I \subset$ int $J \subset(0,1)$,

$$
\begin{gathered}
\frac{\varphi(y)-\varphi(x)}{y-x} \geq \lambda^{+} \text {whenever } y \in J, y>x \text { and } \\
\frac{\varphi(y)-\varphi(x)}{y-x} \leq \lambda^{-} \text {whenever } y \in J, y<x .
\end{gathered}
$$


Then there exist closed intervals $I^{*}, J^{*}, \psi \in B(\varphi, \alpha)$ and $\omega>0$ such that $J^{*} \subset$ int $I,\left|J^{*}\right|<\delta, \psi$ is of type $A\left(\mu^{+}, \mu^{-}, J^{*}, I^{*}, \tau\right)$ and the following condition $(C)$ holds:

(C) If $f \in B(\psi, \omega), x^{*} \in I^{*}$ and $y \in J \backslash J^{*}$, then

$$
\begin{gathered}
\frac{f(y)-f\left(x^{*}\right)}{y-x^{*}} \geq \min \left(\lambda^{+}, \mu^{+}\right)-\delta \text { in the case } y>x^{*} \text { and } \\
\frac{f(y)-f\left(x^{*}\right)}{y-x^{*}} \leq \max \left(\lambda^{-}, \mu^{-}\right)+\delta \text { in the case } y<x^{*} .
\end{gathered}
$$

Proof. It is easy to see that there exists an interval $[c, d] \subset \operatorname{int} I$ such that $x \in(c, d), \varphi$ is linear on $[x, d]$ with a slope $s_{1}^{+} \geq \lambda^{+}$and $\varphi$ is linear on $[c, x]$ with a slope $s_{1}^{-} \leq \lambda^{-}$. Using Lemma 2.4 (for $\gamma:=\delta / 2$ ), we can choose $\xi>0$ such that $[x-\xi, x+\xi] \subset(c, d)$ and, if $x^{*} \in(x-\xi, x+\xi)$ and $X^{*} \in(\varphi(x)-\xi, \varphi(x)+\xi)$, then

$$
\begin{gathered}
\frac{\varphi(y)-X^{*}}{y-x^{*}} \geq \lambda^{+}-\delta / 2 \text { for } y \in J, y \geq d \text { and } \\
\frac{\varphi(y)-X^{*}}{y-x^{*}} \leq \lambda^{-}+\delta / 2 \text { for } y \in J, y \leq c .
\end{gathered}
$$

It is geometrically obvious and easy to prove that there exist closed intervals $I^{*}, J^{*},[u, v]$ with

$$
x \in I^{*} \subset J^{*}=[p, q] \subset(u, v) \subset(x-\xi, x+\xi)
$$

and a piece-wise linear function $\psi \in B(\varphi, \alpha)$ of type $A\left(\mu^{+}, \mu^{-}, J^{*}, I^{*}, \tau\right)$ such that $\psi$ is linear with a slope $s_{2}^{+} \geq \lambda^{+}$on $[q, v], \psi$ is linear with a slope $s_{2}^{-} \leq \lambda^{-}$ on $[u, p], \varphi(t)=\psi(t)$ for $t \in[0,1] \backslash(u, v)$ and

$$
\psi\left(I^{*}\right) \subset(\varphi(x)-\xi, \varphi(x)+\xi) .
$$

By Lemma 2.5, choose $\omega>0$ such that the following holds:

$$
\begin{aligned}
& \text { If } f \in B(\psi, \omega), x^{*} \in I^{*} \text { and } y \in[0,1] \backslash J^{*} \text {, then } \\
& \left|\frac{f(y)-f\left(x^{*}\right)}{y-x^{*}}-\frac{\psi(y)-\psi\left(x^{*}\right)}{y-x^{*}}\right|<\frac{\delta}{2} .
\end{aligned}
$$

To prove the condition (C), suppose that $f \in B(\psi, \omega), x^{*} \in I^{*}$ and $y \in$ $J \backslash J^{*}, y>x^{*}$ are given. 
If $y>d$, then (7), (6) and (5) (used for $X^{*}:=\psi\left(x^{*}\right)$ ) imply

$$
\frac{f(y)-f\left(x^{*}\right)}{y-x^{*}}>\frac{\psi(y)-\psi\left(x^{*}\right)}{y-x^{*}}-\frac{\delta}{2}=\frac{\varphi(y)-\psi\left(x^{*}\right)}{y-x^{*}}-\frac{\delta}{2} \geq \lambda^{+}-\delta .
$$

If $x^{*}<y \leq d$, then observe that $\psi_{+}^{\prime}(t) \geq \min \left(\lambda^{+}, \mu^{+}\right)$for each $t \in\left[x^{*}, d\right]$. This fact and (7) give

$$
\frac{f(y)-f\left(x^{*}\right)}{y-x^{*}} \geq \frac{\psi(y)-\psi\left(x^{*}\right)}{y-x^{*}}-\frac{\delta}{2} \geq \min \left(\lambda^{+}, \mu^{+}\right)-\frac{\delta}{2} .
$$

Thus we have proved the first part of condition $(\mathrm{C})$. The second part can be proved in a symmetrical way.

In the following, we will use the Banach-Mazur game in a metric space. By this we mean the following infinite game between two players:

Let $X$ be a metric space and let $Q \subset X$ be given. In the first step the first player chooses an open ball $B\left(f_{1}, \alpha_{1}\right)$. In the second step the second player chooses an open ball $B\left(g_{1}, \beta_{1}\right) \subset B\left(f_{1}, \alpha_{1}\right)$, in the third step the first player chooses an open ball $B\left(f_{2}, \alpha_{2}\right) \subset B\left(g_{1}, \beta_{1}\right)$, and so on. If

$$
\bigcap_{n=1}^{\infty} B\left(g_{n}, \beta_{n}\right) \subset Q
$$

then the second player wins. In the opposite case the first player wins. We shall need the following theorem.

Theorem 2.7. ([O1]) The second player has a winning strategy in the BanachMazur game if and only if $Q$ is a residual subset of $X$ (i.e., $X \backslash Q$ is a first category set).

A proof of this theorem can be found in [O2] in the case $X=(0,1)$; the proof in the general situation (cf. [O1]) is essentially the same.

To make the proof of Theorem 1.4 clearer, we will prove first the following proposition, from which we then easily deduce Theorem 1.4.

Proposition 2.8. Let $-\infty \leq D_{+} \leq D^{-} \leq \infty$ be extended real numbers. Then, for a typical $f \in \mathcal{C}$, there exists $x \in(0,1)$ such that $D_{+}=D_{+} f(x)=$ $\underline{D}_{a p}^{+} f(x)$ and $D^{-}=D^{-} f(x)=\bar{D}_{a p}^{-} f(x)$.

PROOF. We can clearly choose sequences of real numbers $\left(\lambda_{n}^{+}\right)_{1}^{\infty},\left(\lambda_{n}^{-}\right)_{1}^{\infty}$ such that

$$
\lambda_{n}^{+}<\lambda_{n}^{-}, \lambda_{n}^{+} \rightarrow D_{+}, \lambda_{n}^{-} \rightarrow D^{-}
$$


Denote by $Q$ the set of all $f \in \mathcal{C}$ for which there exists $x \in(0,1)$ such that $D_{+}=D_{+} f(x)=\underline{D}_{a p}^{+} f(x)$ and $D^{-}=D^{-} f(x)=\bar{D}_{a p}^{-} f(x)$. By Theorem 2.7 it is sufficient to describe a winning strategy for the second player in the Banach-Mazur game for $X:=\mathcal{C}$ and $Q$.

By our strategy the second player will construct in his $p$ th move not only a ball $B\left(g_{p}, \beta_{p}\right)$ such that

$$
B\left(g_{p}, \beta_{p}\right) \subset B\left(f_{p}, \alpha_{p}\right)
$$

but also closed intervals $I_{p}, J_{p}$ such that the following conditions hold:

$$
\begin{gathered}
I_{p} \subset \text { int } J_{p} \subset(0,1),\left|J_{p}\right|<1 / p \text { and, if } p \geq 2, J_{p} \subset \text { int } I_{p-1} ; \\
\text { If } p \geq 2, x \in I_{p} \text { and } f \in B\left(g_{p}, \beta_{p}\right), \text { then there exist } \\
\text { numbers } 0<h^{+} \leq 1 / p, 0<h^{-} \leq 1 / p \text { such that } \\
\left|\left\{y \in\left(x, x+h^{+}\right):\left|\frac{f(y)-f(x)}{y-x}-\lambda_{p}^{+}\right|<\frac{1}{p}\right\}\right| \geq \frac{h^{+}}{2} \text { and } \\
\left|\left\{y \in\left(x-h^{-}, x\right):\left|\frac{f(y)-f(x)}{y-x}-\lambda_{p}^{-}\right|<\frac{1}{p}\right\}\right| \geq \frac{h^{-}}{2} ; \\
\text { If } p \geq 2, x \in I_{p}, f \in B\left(g_{p}, \beta_{p}\right) \text { and } y \in J_{p-1} \backslash J_{p}, \quad \text { then } \\
\frac{f(y)-f(x)}{y-x} \geq \min \left(\lambda_{p}^{+}, \lambda_{p-1}^{+}\right)-\frac{1}{p} \text { for } y>x \text { and } \\
\frac{f(y)-f(x)}{y-x} \leq \max \left(\lambda_{p}^{-}, \lambda_{p-1}^{-}\right)+\frac{1}{p} \text { for } y<x ;
\end{gathered}
$$

For each $f \in B\left(g_{p}, \beta_{p}\right)$ there exists a point $x \in \operatorname{int} I_{p}$ such that

$$
\begin{gathered}
\frac{f(y)-f(x)}{y-x} \geq \lambda_{p}^{+} \text {for } y \in J_{p}, y>x \text { and } \\
\frac{f(y)-f(x)}{y-x} \leq \lambda_{p}^{-} \text {for } y \in J_{p}, y<x .
\end{gathered}
$$

First we will prove that the second player can play according to this strategy in all moves.

Thus suppose that, for a fixed natural number $n$, the second player has played $n-1$ moves and the first player has played $n$ moves such that the conditions (9)-(13) hold for each $p<n$. We know that $B\left(f_{n}, \alpha_{n}\right) \subset B\left(g_{n-1}, \beta_{n-1}\right)$ 
(if $n>1$ ) and we can clearly suppose without any loss of generality that $f_{n}$ is a piece-wise linear function. Our task is to construct $g_{n}, \beta_{n}, I_{n}, J_{n}$ such that the conditions (9)-(13) hold for $p=n$.

First consider the case $n=1$. Then the conditions (11), (12) are trivially satisfied for $p=n=1$. We can clearly find intervals $I_{1}, J_{1}$ such that $(10)$ holds for $p=1$ and a function $g_{1} \in B\left(f_{1}, \alpha_{1}\right)$ of type $A\left(\lambda_{1}^{+}, \lambda_{1}^{-}, J_{1}, I_{1}, 1\right)$. Now choose $\beta_{1}>0$ so small, that (9) holds for $p=1$ and $\beta_{1}<(1 / 8)\left(\lambda_{1}^{-}-\lambda_{1}^{+}\right)\left|I_{1}\right|$. Then Lemma 2.2 implies that also (13) holds for $p=1$.

Now consider the case $n \geq 2$. Since $f_{n} \in B\left(g_{n-1}, \beta_{n-1}\right)$ and (13) holds for $p=n-1$, we can choose $x \in \operatorname{int} I_{n-1}$ such that

$$
\begin{gathered}
\frac{f_{n}(y)-f_{n}(x)}{y-x} \geq \lambda_{n-1}^{+} \text {for } y \in J_{n-1}, y>x \text { and } \\
\frac{f_{n}(y)-f_{n}(x)}{y-x} \leq \lambda_{n-1}^{-} \text {for } y \in J_{n-1}, y<x .
\end{gathered}
$$

Thus the assumptions of Lemma 2.6 are satisfied for

$$
\begin{gathered}
\lambda^{+}:=\lambda_{n-1}^{+}, \lambda^{-}:=\lambda_{n-1}^{-}, \mu^{+}:=\lambda_{n}^{+}, \mu^{-}:=\lambda_{n}^{-}, x, \delta:=1 / n, \\
\tau:=\frac{1}{2 n\left(\lambda_{n}^{-}-\lambda_{n}^{+}\right)}, \alpha:=\alpha_{n}, I:=I_{n-1}, J:=J_{n-1} \text { and } \varphi:=f_{n} .
\end{gathered}
$$

Therefore we can choose intervals

$$
I^{*}=: I_{n}, J^{*}=: J_{n}, \psi=: g_{n} \in B\left(f_{n}, \alpha_{n}\right) \text { and } \omega>0
$$

such that $J_{n} \subset \operatorname{int} I_{n-1},\left|J_{n}\right|<1 / n, g_{n}$ is of type $A\left(\lambda_{n}^{+}, \lambda_{n}^{-}, J_{n}, I_{n}, \tau\right)$ and the condition (C) of Lemma 2.6 holds. Now choose $0<\beta_{n}<\omega$ so small that

$$
\frac{4 \beta_{n}}{\left|I_{n}\right|}<\frac{1}{2 n}, \beta_{n}<\frac{1}{8}\left(\lambda_{n}^{-}-\lambda_{n}^{+}\right)\left|I_{n}\right| \text { and } B\left(g_{n}, \beta_{n}\right) \subset B\left(f_{n}, \alpha_{n}\right) .
$$

We see that (9) and (10) hold for $p=n$. Since $\beta_{n}<\omega$, the condition (C) of Lemma 2.6 implies that (12) holds for $p=n$.

Since $\tau\left(\lambda_{n}^{-}-\lambda_{n}^{+}\right)+4 \beta_{n} /\left|I_{n}\right|<1 / n$ and $\left|J_{n}\right|<1 / n$, the application of Lemma 2.3 to

$$
g:=g_{n}, \lambda^{+}:=\lambda_{n}^{+}, \lambda^{-}:=\lambda_{n}^{-}, J:=J_{n}, I:=I_{n}, \tau, \beta:=\beta_{n}
$$

gives the validity of (11) for $p=n$.

Lemma 2.2 immediately implies that (13) holds for $p=n$.

To prove that the described strategy is a winning one, suppose that a game in which the second player used this strategy is over and $f \in \bigcap_{n=1}^{\infty} B\left(g_{n}, \beta_{n}\right)$ is given. By (10) there exists $x \in \bigcap_{n=1}^{\infty} I_{n} \subset(0,1)$. 
Now we prove the main inequality $D_{+} f(x) \geq D_{+}$. In the opposite case we can choose $D_{+} f(x)<q<D_{+}$. By (8) there exists $n_{0}$ such that $\lambda_{n}^{+}>q+1 / n$ for $n \geq n_{0}$. Now consider an arbitrary $y>x, y \in \operatorname{int} J_{n_{0}}$. By condition (10) we can clearly find $p \geq n_{0}+1$ such that $y \in J_{p-1} \backslash J_{p}$. Then we obtain by (12)

$$
\frac{f(y)-f(x)}{y-x} \geq \min \left(\lambda_{p}^{+}, \lambda_{p-1}^{+}\right)-\frac{1}{p} \geq q,
$$

which contradicts $q>D_{+} f(x)$, since $x \in \operatorname{int} J_{n_{0}}$.

Since the conditions (8) and (11) easily imply $D_{+} \geq \underline{D}_{a p}^{+} f(x)$, we obtain $D_{+}=D_{+} f(x)=\underline{D}_{a p}^{+} f(x)$.

The equations $D^{-}=D^{-} f(x)=\bar{D}_{a p}^{-} f(x)$ are proved in a symmetrical way. Thus $f \in Q$, which completes the proof.

Now we are ready to prove Theorem 1.4.

Proof of Theorem 1.4. If

$$
D^{+}=D^{-}=\infty \text { and } D_{+}=D_{-}=-\infty
$$

then the conclusion of the theorem immediately follows from Jarník's result [J4] which says that, for a typical $f \in \mathcal{C}$, almost all $x \in(0,1)$ are essential knot points of $f$ (for an account on improvements of this Jarník's result see $[\mathrm{Z} 1])$.

If the assumption (a) of Theorem 1.4 holds but (14) does not hold, then

$$
\text { either } D_{+} \neq-\infty \text { or } D^{-} \neq \infty \text {. }
$$

Combining Proposition 2.8 and Theorem 1.3 (and the fact that the intersection of two residual sets is residual), we see that for a typical $f \in \mathcal{C}$ there exists a point $x \in(0,1)$ for which $D_{+} f(x)=\underline{D}_{a p}^{+} f(x)=D_{+}, D^{-} f(x)=\bar{D}_{a p}^{-} f(x)=$ $D^{-}$and either $(\alpha)$ or $(\beta)$ of Theorem 1.3 holds. Therefore by (15)

$$
\bar{D}_{a p}^{+} f(x)=\infty=D^{+}, \underline{D}_{a p}^{-} f(x)=-\infty=D_{-}
$$

and the conclusion of Theorem 1.4 follows.

The case when (b) holds, but (14) does not hold, is quite symmetrical. It can be easily reduced to the preceding case using the fact that the mapping $f \rightarrow-f$ is a homeomorphism on $\mathcal{C}$ and relations between derivates of $f$ and $-f$. 


\section{References}

[B] S. Banach, Über die Bairesche Kategorie gewisser Funktionenmengen, Studia Math. 3 (1931), 174-179.

[G1] K. M. Garg, On a residual set of continuous functions, Czechoslovak Math. J. 20(95), (1970), 537-543.

[G2] K. M. Garg, Theory of Differentiation, John Willey, New York, 1998.

[J1] V. Jarník, Über die Differenzierbarkeit stetiger Funktionen, Fund. Math. 21 (1933), 48-58.

[J2] V. Jarník, Sur la dérivabilité des fonctions continues, Publications de la Fac. des. Sc. de L'Univ. Charles 129 (1934), 9 pp.

[J3] V. Jarník, Sur la dérivée approximative unilatérale, Věstník Král. Čes. Spol. Nauk. Tř. II. Roč. 1934, 10 pp.

[J4] V. Jarník, Sur les nombres dérivés approximatifs, Fund. Math. 22 (1934), $4-16$.

[M] S. Mazurkiewicz, Sur les fonctions non dérivables, Studia Math. 3 (1931), 92-94.

[O1] J. Oxtoby, The Banach-Mazur game and Banach category theorem, in: Contributions to the Theory of Games III, Ann. of Math. Stud. 39 (1957), 159-163.

[O2] J. Oxtoby, Measure and category, Springer-Verlag, New York, 1980.

[S] S. Saks, On the functions of Besicovitch in the space of continuous functions, Fund. Math. 19 (1932), 211-219.

[Z1] L. Zajíček, The differentiability structure of typical functions in $C(0,1)$, Real Anal. Exchange 13(1987/88), pp. 119, 113-116, 93.

[Z2] L. Zajíček, On essential derived numbers of typical continuous functions, Tatra Mountains Math. Publ. 2(1993), 1-3. 